\title{
On the Two-Moment Approximation of the Discrete-Time GI/G/1 Queue with a Single Vacation
}

\author{
Doo Ho Lee \\ Department of Industrial and Management Engineering, Kangwon National University, 346 Joongang-ro, \\ Samecheok-si, Gangwon-do 29513, Republic of Korea \\ Correspondence should be addressed to Doo Ho Lee; enjdhlee@gmail.com
}

Received 9 July 2016; Revised 8 September 2016; Accepted 28 September 2016

Academic Editor: Juan R. Torregrosa

Copyright (C) 2016 Doo Ho Lee. This is an open access article distributed under the Creative Commons Attribution License, which permits unrestricted use, distribution, and reproduction in any medium, provided the original work is properly cited.

We consider a discrete-time GI/G/1 queue in which the server takes exactly one vacation each time the system becomes empty. The interarrival times of arriving customers, the service times, and the vacation times are all generic discrete random variables. Under our study, we derive an exact transform-free expression for the stationary system size distribution through the modified supplementary variable technique. Utilizing obtained results, we introduce a simple two-moment approximation for the system size distribution. From this, approximations for the mean system size along with the system size distribution could be obtained. Finally, some numerical examples are given to validate the proposed approximation method.

\section{Introduction}

For several decades, discrete-time queues with the various vacation policies have been receiving growing attention due to their applications to a variety of time-slotted digital communication systems and other synchronous systems. This is due to the fact that discrete-time queues are more suitable for modeling those systems where basic operational units are bits, packets, and cells. On the other hand, server vacation models are characterized by utilizing the idle time of the server to do other work, such as maintenance work, servicing secondary customers, machine repair, or just taking a break. Doshi [1] gives a large number of examples, as does Takagi [2].

Discrete-time queues where the interarrival time, the service time, and the vacation times have general distributions present an interesting subject with which to develop a practical analysis method. Generally, however, the analysis of such a queue is notoriously difficult due to the limited information on their distributions. While several approximations have been proposed, they are often computationally demanding. Moreover, most approximation methods have been applied to continuous-time queues.

In this paper, we consider the discrete-time GI/G/1 queue, in which the server takes exactly one vacation each time the system empties. Upon returning to the system after a vacation, the server begins to serve a customer if any has arrived during a vacation. If none has arrived, the server will wait until a customer arrives. For this discrete-time GI/G/1/SV queue under an exhaustive first-in first-out service discipline, where SV stands for single vacation, we derive an exact transform-free expression of the steady state system size (or queue length) distribution through a modified supplementary variable technique (modified SVT). The first step of the modified SVT is to define a discrete-time Markov chain by including appropriate supplementary variables into the state vector. The second step is to construct the steady state system balance equations. The last step is to solve these equations by directly summing each equation after multiplying a supplement variate. The result thus obtained is the steady state system size distribution expressed as not probability generating functions but conditional expectations. In other words, we derive an exact transform-free expression for the steady state system size distribution.

There are several studies on the discrete-time queues with a single vacation. A discrete-time Geo/G/1/SV queue was considered by Takagi [3], who obtains the probability generating functions (PGFs) of the system size distribution at an arbitrary epoch and waiting time distribution using a stochastic decomposition property. For a discrete-time 
GI/Geo/1/GSV, where GSV stands for geometric single vacation, Chae et al. [4] derived the PGFs of the system size distribution at arrival epochs, at departure epochs, and at arbitrary epochs through an embedded Markov chain and a semi-Markov process. They also verify the decomposition structure of the PGF of the waiting time distribution. Fiems and Bruneel [5] consider a discrete-time GI/G/1 queue with modified multiple vacations, called timed vacations, in which vacations occur whenever the queue becomes empty or whenever a timer geometrically distributed expires. Using the PGF approach, a variety of performance measures are derived.

Addressing the continuous-time queues, Kempa [6] analyzes a continuous-time GI/G/1 queue with batch arrival of customers and a single exponential vacation. In [6], the author applies a technique of integral equations to obtain the Laplace transform (LT) of the joint distribution of three random variables: the first busy period, the first vacation period, and the number of customers served during the first busy period.

All of the aforementioned studies give the transformed results because the main analysis approaches are based on transformation techniques such as PGF and LT. The only study that obtains transform-free results can be found by Chae et al. [7]. They first propose a modified SVT and apply it to a continuous-time GI/G/1/K queue with multiple vacations. However, to the best of our knowledge, studies of a discrete-time queue with general interarrival times, general service times, and general vacation times cannot be found. This motivates us to analyze the GI/G/1/SV in a discrete-time environment.

This paper is organized as follows. In Section 2, we introduce the modified SVT briefly and present the transformfree system size distribution for a discrete-time GI/G/1/SV. In Section 3, we propose a simple approximation, termed here a two-moment approximation, for the system size distribution. A two-moment approximation for a continuoustime queue has been reported in the literature $[8,9]$, but there is no precedent for a discrete-time queue. In Section 4, numerical experiments are conducted to demonstrate that our approximations are remarkably simple yet provide fairly good performance.

\section{The Steady State Queue Length Distribution of a GI/G/1 Queue with Single Vacation}

We adopt the late arrival system with delayed access (LASDA) model [3]. Let the time axis be marked by $t=0,1,2, \ldots$. According to the LAS-DA model, a potential customer arrival takes place during interval $\left(t^{-}, t\right)$ and a potential service completion occurs during interval $\left(t, t^{+}\right)$, where $t^{+}$and $t^{-}$ represent $\lim _{\Delta t \rightarrow 0}(t+|\Delta t|)$ and $\lim _{\Delta t \rightarrow 0}(t-|\Delta t|)$, respectively. The GI/G/1/SV queue is assumed to operate as follows. Suppose that a customer departs the system during interval $\left(t, t^{+}\right)$leaving behind no customers in the system. The single server then begins to take a single vacation at $t^{+}$. Suppose that the length of the vacation that begins at $t^{+}$is equal to $u, u=$ $1,2, \ldots$, and this vacation will end at $x$, where $x=t^{+}+u$. Upon returning to the system at $x$, the server will begin to serve a customer at $x^{-}$if any has arrived during interval $\left(t^{+}, x^{-}\right)$. If none has arrived, the server will wait until a customer arrives in the system without taking another vacation.

Interarrival times $A$ are independent and identically distributed (iid) discrete random variables (RVs) that have the following distribution: $\operatorname{Pr}\{A=i\}=a_{i}, i=1,2, \ldots$. Vacation times $V$ are iid discrete RVs and have the following distribution: $\operatorname{Pr}\{V=j\}=v_{j}, j=1,2, \ldots$ Service times $S$ are iid discrete RVs and have the following distribution: $\operatorname{Pr}\{S=$ $k\}=s_{k}, k=1,2, \ldots$. We assume that interarrival times, vacation times, and service times are mutually independent. Let $N\left(t^{-}\right)$denote the number of customers in the system at $t^{-}$ and define

$$
\xi\left(t^{-}\right)= \begin{cases}0, & \text { The server is taking a vacation at } t^{-}, \\ 1, & \text { The server is available at } t^{-} .\end{cases}
$$

Then, $\left\{N\left(t^{-}\right), \xi\left(t^{-}\right), A_{R}\left(t^{-}\right), S_{R}\left(t^{-}\right), V_{R}\left(t^{-}\right), t=1,2, \ldots\right\}$ becomes the discrete-time Markov chain, where the supplementary variables $A_{R}\left(t^{-}\right), S_{R}\left(t^{-}\right)$, and $V_{R}\left(t^{-}\right)$, respectively, denote the remaining interarrival time, the remaining service time, and the remaining vacation time all at $t^{-}$. Let us define the following probabilities:

$$
\begin{aligned}
& Q_{n}(i, j)=\lim _{t \rightarrow \infty} \operatorname{Pr}\left\{N\left(t^{-}\right)=n, \xi\left(t^{-}\right)=0, A_{R}\left(t^{-}\right)\right. \\
& \left.=i, V_{R}\left(t^{-}\right)=j\right\}, \quad n \geq 0, i, j=0,1, \ldots, \\
& P_{0}(i)=\lim _{t \rightarrow \infty} \operatorname{Pr}\left\{N\left(t^{-}\right)=0, \xi\left(t^{-}\right)=1, A_{R}\left(t^{-}\right)=i\right\}, \\
& i=0,1, \ldots, \\
& P_{n}(i, k)=\lim _{t \rightarrow \infty} \operatorname{Pr}\left\{N\left(t^{-}\right)=n, \xi\left(t^{-}\right)=1, A_{R}\left(t^{-}\right)\right. \\
& \left.=i, S_{R}\left(t^{-}\right)=k\right\}, \quad n \geq 1, i, k=0,1, \ldots, \\
& Q_{n}=\sum_{i=0}^{\infty} \sum_{j=0}^{\infty} Q_{n}(i, j), \quad n \geq 0, \\
& P_{0}=\sum_{i=0}^{\infty} P_{0}(i) \\
& P_{n}=\sum_{i=0}^{\infty} \sum_{k=0}^{\infty} P_{n}(i, k), \quad n \geq 1, \\
& \pi_{n}=Q_{n}+P_{n}, \quad n \geq 0 .
\end{aligned}
$$

Here, $\pi_{n}$ refers to the probability that $n$ customers are in the system at an arbitrary time. Considering mutually exclusive events that can occur during one slot, the system balance equations for the discrete-time GI/G/1/SV queue are given by

$$
\begin{aligned}
& Q_{0}(i, j)=Q_{0}(i+1, j+1)+P_{1}(i+1,0) v_{j+1}, \\
& Q_{n}(i, j)=Q_{n}(i+1, j+1)+Q_{n-1}(0, j+1) a_{i+1},
\end{aligned}
$$




$$
\begin{aligned}
P_{0}(i)= & P_{0}(i+1)+Q_{0}(i+1,0) \\
P_{1}(i, k)= & P_{1}(i+1, k+1) \\
& +\left\{Q_{1}(i+1,0)+P_{2}(i+1,0)\right\} s_{k+1} \\
& +\left\{Q_{0}(0,0)+P_{0}(0)+P_{1}(0,0)\right\} a_{i+1} s_{k+1}, \\
P_{n}(i, k)= & P_{n}(i+1, k+1)+P_{n-1}(0, k+1) a_{i+1} \\
& +\left\{Q_{n}(i+1,0)+P_{n+1}(i+1,0)\right\} s_{k+1} \\
& +\left\{Q_{n-1}(0,0)+P_{n}(0,0)\right\} a_{i+1} s_{k+1},
\end{aligned}
$$

$n \geq 2$.

The left-hand sides of (3a), (3b), and (3c) represent the probabilities of the system state at $(t+1)^{-}$in a steady state. The right-hand sides of (3a), (3b), and (3c) are then expressed in terms of the probabilities of the system state at $t^{-}$in a steady state, together with the probabilities of all potential queueing activities that can occur during $\left(t^{-}, t^{+}\right)$. Note that $P_{n}(0,0)\left(Q_{n}(0,0)\right)$ has a positive value since an arrival and a departure can occur simultaneously (since an arrival and vacation termination can occur simultaneously) in the discrete-time setting.

In the modified SVT, we first sum (3a) both over $i$ and $j, 0 \leq i, j \leq \infty$ and sum (3b) both over $i, 0 \leq i \leq \infty$. We also sum (3c) both over $i$ and $k, 0 \leq i, k \leq \infty$. Secondly, we multiply $i+1$ to both sides of (3a), (3b), and (3c) and sum over $i, j$, and $k, 0 \leq i, j, k \leq \infty$. Finally, we multiply $j+1$ to both sides of (3a) and sum both over $i$ and $j, 0 \leq i, j \leq \infty$, and then multiply $k+1$ to both sides of (3c) and sum both over $i$ and $k$, $0 \leq i, k \leq \infty$. This procedure is then applied to our queuing model. We first sum (3a) both over $i$ and $j, 0 \leq i, j \leq \infty$ and sum (3b) both over $i, 0 \leq i \leq \infty$. We also sum (3c) both over $i$ and $k, 0 \leq i, k \leq \infty$. Simplifying the results (for more detailed derivation, see Appendix A), we get

$$
\begin{aligned}
P_{0}(0) & =\sum_{i=1}^{\infty} Q_{0}(i, 0), \\
\sum_{i=1}^{\infty} P_{1}(i, 0) & =\sum_{j=0}^{\infty} Q_{0}(0, j)+\sum_{i=1}^{\infty} Q_{0}(i, 0), \\
\sum_{i=1}^{\infty} P_{n}(i, 0) & =\sum_{j=0}^{\infty} Q_{n-1}(0, j)+\sum_{k=1}^{\infty} P_{n-1}(0, k), \quad n \geq 2, \\
\sum_{i=1}^{\infty} Q_{n}(i, 0) & =\sum_{j=1}^{\infty} Q_{n-1}(0, j)-\sum_{j=0}^{\infty} Q_{n}(0, j), \quad n \geq 1 .
\end{aligned}
$$

Clearly, since we do not consider the situation where customers balk or abandon their services, the departure rate (output rate) of customers is identical to the arrival rate (input rate) $\lambda=1 / E[A]$. Since the system is equipped with a single server, the server utilization (or the probability that the server is busy), denoted by $\rho$, is equal to $\rho=\lambda E[S]$. For the system to be stable, we assume that $\rho<1$.
Let $Q_{n}^{A}\left(P_{n}^{A}\right)$ denote the probability that an arriving customer sees $n$ customers when the server is on vacation (when the server is available). This gives

$$
\begin{aligned}
Q_{n}^{A} & =\frac{\sum_{j=0}^{\infty} Q_{n}(0, j)}{\lambda}, \quad n \geq 0, \\
P_{0}^{A} & =\frac{P_{0}(0)}{\lambda}=\frac{\sum_{i=1}^{\infty} Q_{0}(i, 0)}{\lambda}, \\
P_{n}^{A} & =\frac{\sum_{k=0}^{\infty} P_{n}(0, k)}{\lambda}, \quad n \geq 1 .
\end{aligned}
$$

Remark 1. In (5a), $\sum_{j=0}^{\infty} Q_{n}(0, j)$ can be interpreted as the rate (or the expected frequency per unit time) that an arriving customer sees $n$ customers when the server is on vacation. Since $\lambda$ is the expected number of arrivals per unit time and $Q_{n}^{A}$ is the probability that an arriving customer sees $n$ customers when the server is on vacation, we have the concrete result: $\lambda Q_{n}^{A}=\sum_{j=0}^{\infty} Q_{n}(0, j)$. The rest of equations in (5a), (5b), and (5c) can be obtained by the same manner.

Likewise, let $\pi_{n}^{D}$ denote the probability that a departing customer leaves behind $n$ customers. The result is then

$$
\begin{aligned}
& \pi_{0}^{D}=\frac{\sum_{i=1}^{\infty} P_{1}(i, 0)}{\lambda}, \\
& \pi_{n}^{D}=\frac{P_{n}(0,0)+\sum_{i=1}^{\infty} P_{n+1}(i, 0)}{\lambda}, \quad n \geq 0 .
\end{aligned}
$$

Remark 2. $P_{n}(0,0)+\sum_{i=1}^{\infty} P_{n+1}(i, 0)$ can be interpreted as the rate (or the expected frequency per unit time) that a departing customer leaves behind $n$ customers in the system. Since $\lambda$ is also the expected number of departures per unit time and $\pi_{n}^{D}$ is the probability that a departing customer leaves behind $n$ customers in the system, $\lambda \pi_{n}^{D}=P_{n}(0,0)+\sum_{i=1}^{\infty} P_{n+1}(i, 0)$ should hold. Note that (5a), (5b), (5c), (6a), and (6b) follow from the renewal reward theorem [10].

Substituting (5a), (5b), (5c), (6a), and (6b) into (4a) and (4b), we identify (4a) as $\lambda Q_{n}^{A}+\lambda P_{n}^{A}=\lambda \pi_{n}^{D}$. Thus, we have the following Burke's theorem in the discrete-time GI/G/1/SV queue:

$$
Q_{n}^{A}+P_{n}^{A}=\pi_{n}^{D}, \quad n \geq 0 .
$$

Next, we multiply $i+1$ to both sides of (3a), (3b), and (3c) and sum over $i, j$, and $k, 0 \leq i, j, k \leq \infty$. Simplifying the results, we obtain

$$
\begin{aligned}
Q_{0}= & \sum_{i=1}^{\infty} i P_{1}(i, 0)-\sum_{i=1}^{\infty} i Q_{0}(i, 0), \\
Q_{n}= & \sum_{j=0}^{\infty} Q_{n-1}(0, j) E[A] \\
& -\left\{\sum_{i=1}^{\infty} i Q_{n}(i, 0)+Q_{n-1}(0,0) E[A]\right\},
\end{aligned}
$$




$$
\begin{aligned}
P_{0}= & \sum_{i=1}^{\infty} i Q_{0}(i, 0), \\
P_{1}= & P_{0}(0) E[A]+\left\{\sum_{i=1}^{\infty} i P_{2}(i, 0)+P_{1}(0,0) E[A]\right\} \\
& -\sum_{i=1}^{\infty} i P_{1}(i, 0) \\
& +\left\{\sum_{i=1}^{\infty} i Q_{1}(i, 0)+Q_{0}(0,0) E[A]\right\}, \\
P_{n}= & \left\{\sum_{i=1}^{\infty} i P_{n+1}(i, 0)+P_{n}(0,0) E[A]\right\} \\
& -\left\{\sum_{i=1}^{\infty} i P_{n}(i, 0)+P_{n-1}(0,0) E[A]\right\} \\
& +\sum_{k=0}^{\infty} P_{n-1}(0, k) E[A] \\
& +\left\{\sum_{i=1}^{\infty} i Q_{n}(i+1,0)+Q_{n-1}(0,0) E[A]\right\},
\end{aligned}
$$$$
n \geq 2 \text {. }
$$

Then, we multiply $j+1$ to both sides of (3a) and sum over $i$ and $j, 0 \leq i, j \leq \infty$. Simplifying the results gives

$$
\begin{aligned}
& Q_{0}=\sum_{i=1}^{\infty} P_{1}(i, 0) E[V]-\sum_{j=1}^{\infty} j Q_{0}(0, j), \\
& Q_{n}=\sum_{j=1}^{\infty} j Q_{n-1}(0, j)-\sum_{j=1}^{\infty} j Q_{n}(0, j), \quad n \geq 1 .
\end{aligned}
$$

Finally, we multiply $k+1$ to both sides of (3c) and sum over $i$ and $k, 0 \leq i, k \leq \infty$. Simplifying the results gives

$$
\begin{aligned}
P_{1}= & P_{0}(0) E[S] \\
& +\left\{\sum_{j=0}^{\infty} Q_{0}(0, j)+\sum_{k=0}^{\infty} P_{1}(0, k)\right\} E[S] \\
& -\sum_{k=1}^{\infty} k P_{1}(0, k), \\
P_{n}= & \left\{\sum_{j=0}^{\infty} Q_{n-1}(0, j)+\sum_{k=0}^{\infty} P_{n}(0, k)\right\} E[S] \\
& +\sum_{k=1}^{\infty} k P_{n-1}(0, k)-\sum_{k=1}^{\infty} k P_{n}(0, k), \quad n \geq 2 .
\end{aligned}
$$

To express (8a), (8b), (8c), (8d), (8e), (9a), (9b), (10a), and (10b) in terms of meaningful identities, we define the following conditional expectations: for $n \geq 0, \sigma_{n}=E\left[S_{n}^{A}\right]$,
$\nu_{n}=E\left[V_{n}^{A}\right], \alpha_{n}^{D}=E\left[A_{n}^{D}\right]$, and $\alpha_{n}^{V}=E\left[A_{n}^{V}\right]$, where $S_{n}^{A}\left(V_{n}^{A}\right)$ is the remaining service time (vacation time) at the arrival epoch of a customer who sees $n$ customers in the system and $A_{n}^{D}\left(A_{n}^{V}\right)$ is the remaining interarrival time at the departure epoch of a customer who leaves behind $n$ customers in the system (at the instance when the server completes its vacations, finding $n$ customers in the system). Naturally, $\sigma_{0}=$ $E[S]$. From the definition of a conditional expectation, $\sigma_{n}$, $\nu_{n}, \alpha_{n}^{D}$, and $\alpha_{n}^{V}$ can be rewritten as follows (for more detailed derivation, see Appendix B):

$$
\begin{aligned}
\sigma_{n} & =\frac{\sum_{k=1}^{\infty} k P_{n}(0, k)}{\lambda P_{n}^{A}}, \quad n \geq 0, \\
\nu_{n} & =\frac{\sum_{j=1}^{\infty} j Q_{n}(0, j)}{\lambda Q_{n}^{A}}, \quad n \geq 0, \\
\alpha_{0}^{D} & =\frac{\sum_{i=1}^{\infty} i P_{1}(i, 0)}{\lambda \pi_{0}^{D}}, \\
\alpha_{n}^{D} & =\frac{P_{n}(0,0) E[A]+\sum_{i=1}^{\infty} i P_{n+1}(i, 0)}{\lambda \pi_{n}^{D}}, \quad n \geq 1, \\
\alpha_{0}^{V} & =\frac{\sum_{i=1}^{\infty} i Q_{0}(i, 0)}{\lambda P_{0}^{A}}, \\
\alpha_{n}^{V} & =\frac{Q_{n-1}(0,0) E[A]+\sum_{i=1}^{\infty} i Q_{n}(i, 0)}{\lambda Q_{n-1}^{A}-\lambda Q_{n}^{A}}, \quad n \geq 1 .
\end{aligned}
$$

Substituting (5a), (5b), (5c), (6a), (6b), (11a), (11b), (11c), (11d), (11e), and (11f) into (8a), (8b), (8c), (8d), and (8e), we obtain the first set of system size distribution equations as follows.

Theorem 3. The first set of system size distribution equations for the discrete-time $G I / G / 1 / S V$ queue is given by

$$
\begin{aligned}
Q_{0}= & \lambda \pi_{0}^{D} \alpha_{0}^{D}-\lambda P_{0}^{A} \alpha_{0}^{V}, \\
Q_{n}= & Q_{n-1}^{A}-\lambda Q_{n-1}^{A} \alpha_{n}^{V}+\lambda Q_{n}^{A} \alpha_{n}^{V}, \quad n \geq 1, \\
P_{0}= & \lambda P_{0}^{A} \alpha_{0}^{V}, \\
P_{n}= & P_{n-1}^{A}+\lambda \pi_{n}^{D} \alpha_{n}^{D}-\lambda \pi_{n-1}^{D} \alpha_{n-1}^{D}+\lambda Q_{n-1}^{A} \alpha_{n}^{V} \\
& -\lambda Q_{n}^{A} \alpha_{n}^{V}, \quad n \geq 1 .
\end{aligned}
$$

Substituting (5a), (5b), (5c), (6a), (6b), (11a), (11b), (11c), (11d), (11e), and (11f) into (9a), (9b), (10a), and (10b), we obtain the second set of system size distribution equations as follows.

Theorem 4. The second set of system size distribution equations for the discrete-time GI/G/1/SV queue is given by

$$
\begin{aligned}
Q_{0} & =\lambda \pi_{0}^{D} E[V]-\lambda Q_{0}^{A} v_{0}, \\
Q_{n} & =\lambda Q_{n-1}^{A} v_{n-1}-\lambda Q_{n}^{A} v_{n}, \quad n \geq 1, \\
P_{0} & =1-\rho-\lambda \pi_{0}^{D} E[V],
\end{aligned}
$$




$$
\begin{aligned}
& P_{1}=\rho Q_{0}^{A}+\rho P_{0}^{A}+\rho P_{1}^{A}-\lambda P_{1}^{A} \sigma_{1}, \\
& P_{n}=\rho Q_{n-1}^{A}+\rho P_{n}^{A}+\lambda P_{n-1}^{A} \sigma_{n-1}-\lambda P_{n}^{A} \sigma_{n}, \quad n \geq 2,
\end{aligned}
$$

where $(13 c)$ is derived by normalization, $\sum_{n=0}^{\infty} \pi_{n}=1$. Consequentially, (12a), (12b), (12c), (12d), (13a), (13b), (13c), (13d), and (13e) lead to the following transform-free expressions for the system size distributions just before an arrival and at an arbitrary time, all in product forms.

Theorem 5. The steady state system size distribution for a discrete-time GI/G/1/SV queue is given by

$$
\begin{aligned}
Q_{0}^{A} & =\left(E[V]-\alpha_{0}^{D}+\alpha_{0}^{V}\right) E[A] \phi_{0}, \\
Q_{n}^{A} & =Q_{0}^{A} \prod_{i=1}^{n} \frac{\lambda_{i}^{V}}{\mu_{i}^{V}}, \quad n \geq 1, \\
P_{0}^{A} & =\left(\alpha_{0}^{D}+\nu_{0}-E[V]\right) E[A] \phi_{0}, \\
P_{n}^{A} & =P_{n-1}^{A} \frac{\lambda_{n}^{B}}{\mu_{n}^{B}}+Q_{n-1}^{A} \varepsilon_{n}, \quad n \geq 1, \\
Q_{0} & =\left\{\alpha_{0}^{D} v_{0}+\alpha_{0}^{V}\left(E[V]-v_{0}\right)\right\} \phi_{0}, \\
Q_{n} & =Q_{n}^{A} \gamma_{n}^{V}, \quad n \geq 1, \\
P_{0} & =\left(\alpha_{0}^{D}+v_{0}-E[V]\right) \alpha_{0}^{V} \phi_{0}, \\
P_{n} & =P_{n}^{A} \gamma_{n}^{B}+Q_{n-1}^{A} \delta_{n}, \quad n \geq 1,
\end{aligned}
$$

where

$$
\begin{aligned}
\phi_{0} & =\frac{1-\rho}{\alpha_{0}^{D} \alpha_{0}^{V}+\left(E[V]+\alpha_{0}^{V}\right) \nu_{0}}, \\
\lambda_{n}^{V} & =\alpha_{n}^{V}+\nu_{n-1}-E[A], \\
\mu_{n}^{V} & =\alpha_{n}^{V}+\nu_{n}, \\
\lambda_{n}^{B} & =\alpha_{n-1}^{D}+\sigma_{n-1}-E[A], \\
\mu_{n}^{B} & =\alpha_{n}^{D}+\sigma_{n}-E[S], \\
\varepsilon_{n} & =\frac{\alpha_{n}^{V}-\alpha_{n}^{D}}{\alpha_{n}^{D}+\sigma_{n}-E[S]} \cdot \frac{\lambda_{n}^{V}+\frac{E[S]+\alpha_{n-1}^{D}-\alpha_{n}^{V}}{\mu_{n}^{V}},}{\alpha_{n}^{D}+\sigma_{n}-E[S]} \\
\gamma_{n}^{V} & =\frac{\alpha_{n}^{V} \nu_{n-1}-\alpha_{n}^{V} \nu_{n}+E[A] \nu_{n}}{\left(\alpha_{n}^{V}+\nu_{n-1}-E[A]\right) E[A]}, \\
\gamma_{n}^{B} & =\frac{\alpha_{n}^{D} \sigma_{n-1}+\left(E[S]-\sigma_{n}\right)\left(\alpha_{n-1}^{D}-E[A]\right)}{\left(\alpha_{n-1}^{D}+\sigma_{n-1}-E[A]\right) E[A]}, \\
\delta_{n}= & \rho-\frac{\sigma_{n-1} \varepsilon_{n} \mu_{n}^{B}}{\lambda_{n}^{B} E[A]} .
\end{aligned}
$$

Proof. For each $n, n \geq 0$, we first equate (12a), (12b), (12c), and (12d) with (13a), (13b), (13c), (13d), and (13e). Then, we solve them simultaneously with (7). As a result, we obtain (14a), (14b), (14c), and (14d). Finally, (15a), (15b), (15c), and (15d) follow after combining (14a), (14b), (14c), and (14d) with either ((12a), (12b), (12c), (12d)) or ((13a), (13b), (13c), (13d), and $(13 \mathrm{e}))$. Note that the system size distributions just after a departure can be also obtained from (7).

Let us remark that (15a), (15b), (15c), and (15d) involve the unknown conditional expectations of supplementary variables. In general, these conditional expectations are not easy to compute, except for some special cases such as a Bernoulli arrival process, geometric service times, or geometric vacation times. However, the availability of such expressions provides a basic idea for developing approximations for various performance measures of practical interest. This is discussed in Sections 3 and 4.

\section{The Two-Moment Approximations and Numerical Results}

Making use of the exact results of the system size distribution given in Section 2, we introduce its two-moment approximation. From this, approximations of the various mean performance measures including the mean system size, the mean sojourn time, and the mean waiting time can be carried out. Among others, we focus on the mean system size, which is of great practical importance. We employ the following approximation scheme:

$$
\begin{array}{ll}
\alpha_{n}^{D} \approx \alpha=\frac{E\left[A^{2}\right]+E[A]}{2 E[A]}, & n \geq 0, \\
\alpha_{n}^{V} \approx \alpha=\frac{E\left[A^{2}\right]+E[A]}{2 E[A]}, & n \geq 0, \\
\sigma_{n} \approx \sigma=\frac{E\left[S^{2}\right]-E[S]}{2 E[S]}, & n \geq 1, \\
\nu_{n} \approx \nu=\frac{E\left[V^{2}\right]-E[V]}{2 E[V]}, & n \geq 0,
\end{array}
$$

where $E\left[Y^{2}\right]$ is the second moment of the discrete RV $Y$ whose distribution function is $F$ and $\left(E\left[Y^{2}\right] \pm E[Y]\right) / 2 E[Y]$ is the mean of the equilibrium distribution of $F$. Recall that $\sigma_{0}=E[S]$.

Remark 6. In our setting, the remaining interarrival time of a customer both at a service completion epoch and at a vacation termination epoch does not contain 0 . In contrast, both the remaining service time and the remaining vacation time at a customer arrival epoch contain 0 . Therefore, from the discrete-time inspection paradox, $\alpha_{n}^{D}\left(\alpha_{n}^{V}\right), \sigma_{n}$, and $\nu_{n}$ can approximate $\alpha, \sigma$, and $\nu$, respectively.

Remark 7. Note that the approximation scheme in (17a), (17b), (17c), and (17d) is exact for the Bernoulli arrivals, geometric service times, and geometric vacation times under the LAS-DA model, respectively, due to the memoryless property of the geometric distribution. Therefore, our approximations 
lead to exact results for the discrete-time Geo/Geo/1/GSV queue. However, for some queues with a non-Bernoulli arrivals, general service times, and general vacation times, conditional expectations in (17a), (17b), (17c), and (17d) cannot be easily calculated due to the absence of the information about the interarrival, service, and vacation time distributions. Hence, the quick and simple approximation is to replace all the conditional expectations with the unconditional counterparts like (17a), (17b), (17c), and (17d). This approximation scheme works fairly well and its numerical examples are given in Section 4. A similar approximation scheme was used for the continuous-time GI/G/1/K queue by Kim and Chae [9] and the continuous-time GI/G/c/K queue by Choi et al. [8].

Making these substitutions, (14a), (14b), (14c), (14d), (15a), (15b), (15c), and (15d) can be rewritten as follows:

$$
\begin{aligned}
& Q_{0}^{A} \approx E[A] E[V] \phi, \\
& Q_{n}^{A} \approx \widetilde{Q}_{0}^{A}\left(\frac{\lambda^{V}}{\mu^{V}}\right)^{n}, \quad n \geq 1, \\
& P_{0}^{A} \approx(\alpha+\nu-E[V]) E[A] \phi, \\
& P_{1}^{A} \approx \widetilde{P}_{0}^{A} \frac{\alpha+E[S]-E[A]}{\mu^{B}}+\widetilde{Q}_{0}^{A} \varepsilon, \\
& P_{n}^{A} \approx \widetilde{P}_{n-1}^{A} \frac{\lambda^{B}}{\mu^{B}}+\widetilde{Q}_{n-1}^{A} \varepsilon, \quad n \geq 2, \\
& Q_{0} \approx E[V] \alpha \phi, \\
& Q_{n} \approx \widetilde{Q}_{n}^{A} \gamma^{V}, \quad n \geq 1, \\
& P_{0} \approx(\alpha+\nu-E[V]) \alpha \phi, \\
& P_{1} \approx \\
& \widetilde{P}_{1}^{A} \frac{\alpha E[S]+(E[S]-\sigma)(\alpha-E[A])}{(\alpha+E[S]-E[A]) E[A]} \\
&+\rho_{0} \widetilde{Q}_{0}^{A}\left\{1-\frac{(\alpha+\sigma-E[S]) \varepsilon}{\alpha+E[S]-E[A]}\right\}, \\
& P_{n} \approx \widetilde{P}_{n}^{A} \gamma^{B}+\widetilde{Q}_{n-1}^{A} \delta, \quad n \geq 2,
\end{aligned}
$$

where

$$
\begin{aligned}
\phi & =\frac{1-\rho}{\alpha^{2}+(E[V]+\alpha) \nu}, \\
\lambda^{V} & =\alpha+\nu-E[A], \\
\mu^{V} & =\alpha+\nu, \\
\lambda^{B} & =\alpha+\sigma-E[A], \\
\mu^{B} & =\alpha+\sigma-E[S], \\
\varepsilon & =\frac{E[S]}{\alpha+\sigma-E[S]}, \\
\gamma^{V} & =\frac{v}{\alpha+\nu-E[A]}, \\
\gamma^{B} & =\frac{\alpha \rho+\sigma-E[S]}{\alpha+\sigma-E[A]}, \\
\delta & =\rho-\frac{\varepsilon \mu^{B} \sigma}{E[A] \lambda^{B}} .
\end{aligned}
$$

$\widetilde{Q}_{n}^{A}$ and $\widetilde{P}_{n}^{A}$ are approximate values for $Q_{n}^{A}$ and $P_{n}^{A}$, respectively. For some queues with a non-Bernoulli arrival process, general service times, and general vacation times, (18a), (18b), (18c), (18d), (18e), (18f), (18g), (18h), (18i), and (18j) may result in negative probabilities. In such a case, these approximate values may be set to zero.

From (17a), (17b), (17c), and (17d), approximations of the mean system size can be obtained. Let $L$ denote the mean system size. From (12a), (12b), (12c), (12d), (13a), (13b), (13c), (13d), and (13e), we have

$$
\begin{aligned}
L= & 1+\sum_{m=1}^{\infty} \sum_{n=m}^{\infty}\left(Q_{n}^{A}+P_{n}^{A}\right)-\sum_{n=0}^{\infty} \lambda\left(Q_{n}^{A}+P_{n}^{A}\right) \alpha_{n}^{D}, \\
L= & \rho \sum_{m=1}^{\infty} \sum_{n=m}^{\infty}\left(Q_{n}^{A}+P_{n}^{A}\right)+\sum_{n=0}^{\infty}\left(\rho Q_{n}^{A}+\lambda Q_{n}^{A} v_{n}\right) \\
& +\lambda \sum_{n=1}^{\infty} P_{n}^{A} \sigma_{n}+\rho P_{0}^{A} .
\end{aligned}
$$

Combining (20a) and (20b) with (17a), (17b), (17c), and (17d) yields

$$
L \approx \frac{\lambda(E[S]+\nu+\rho \alpha) \sum_{n=0}^{\infty} \widetilde{Q}_{n}^{A}+\lambda(\sigma+\rho \alpha) \sum_{n=1}^{\infty} \widetilde{P}_{n}^{A}+\lambda(E[S]+\rho \alpha) \widetilde{P}_{0}^{A}}{1-\rho}-\frac{\rho}{1-\rho}
$$

\section{Numerical Example}

In this section, numerical examples are presented to evaluate the performance of our approximation. We apply the results obtained in Section 3 to queues with a variety of interarrival times, service times, and vacation times, but only a few that exhibit representative information are presented in Figures 1-3. In each figure, the horizontal axis represents the system size and the vertical axis does its probability. In all cases, exact values are calculated by differentiating the PGFs of each system size distribution. $\mathrm{MGeO}_{2}, \mathrm{NB}$, and Pois denote a mixture of two different geometric distributions, 


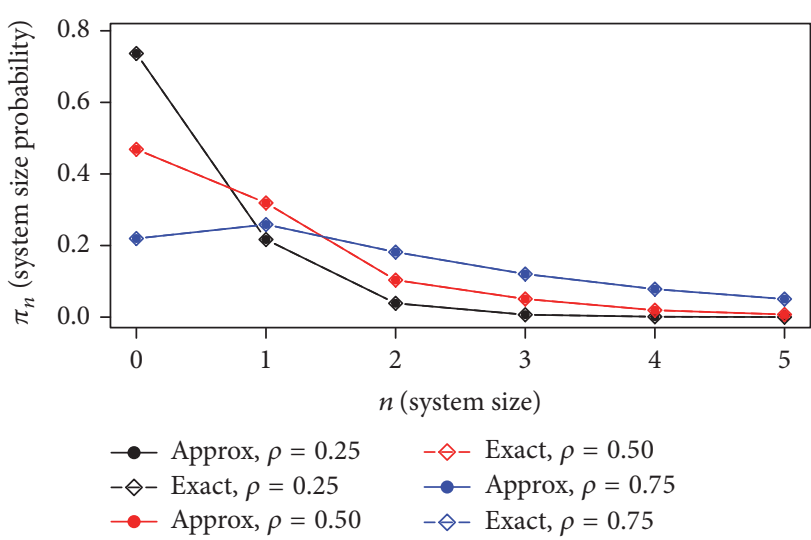

(a) Geo/Geo/1/GSV queue

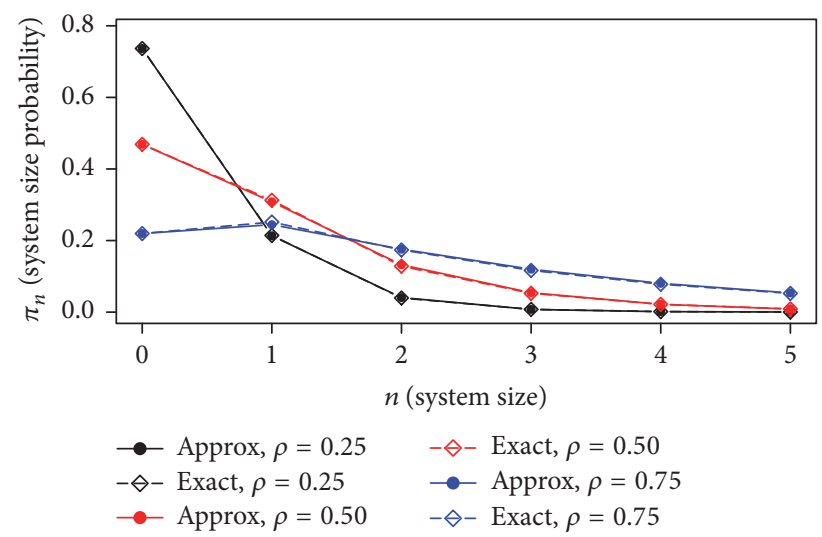

(b) $\mathrm{Geo} / \mathrm{MGeo}_{2} / 1 / \mathrm{GSV}$ queue

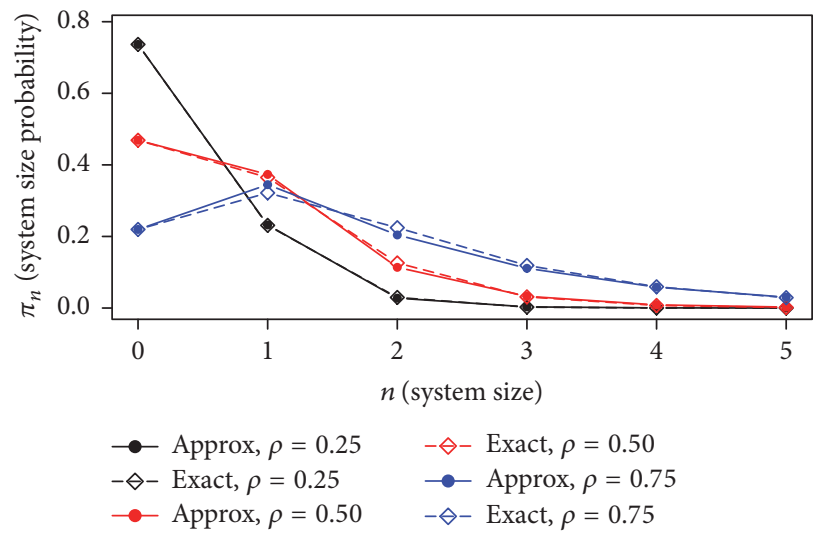

(c) $\mathrm{Geo} / \mathrm{NB} / 1 / \mathrm{GSV}$ queue

Figure 1: Approximation of $\pi_{n}$ in Geo/G/1/GSV queue when $E[S]=2.5$ and $E[V]=2$.

a negative binomial distribution, and a Poisson distribution, respectively.

We compare our approximate results of the system size distribution with exact results for several Bernoulli arrival queues with a GSV in low $(\rho=0.25)$ traffic, in moderate $(\rho=0.50)$ traffic, and in high $(\rho=0.75)$ traffic, as presented in Figure 1. Extensive numerical investigations show that our results are in good agreement with the exact results regardless of the traffic intensities. Figure 2 gives results for Bernoulli arrival queues with a general single vacation. Interestingly, our approximation functions well even though vacation times do not follow geometric distributions.

The approximated and exact values of the system size probability for the non-Bernoulli arrival queues are depicted in Figure 3. In this case, however, the approximation can deteriorate. Thus, one should use our approximation method cautiously for the non-Bernoulli arrival queues. Note that our approximations require only the first two moments of interarrival times, service times, and vacation times. Thus, it is not essential to identify these distributions. The first two moments alone will lead to quick and simple approximate results. We anticipate that our two-moment approximation will be beneficial to those practitioners who seek simple and quick practical answers to queueing systems with a single vacation and other systems.

\section{Concluding Remarks}

For a discrete-time GI/G/1/SV queue, this work presented the exact transform-free expressions of the system size distribution. Then, we proposed the simple two-moment approximation of the system sized distribution and the mean system size. It is worth noting that the modified SVT is basically the same as the conventional SVT except for the last step, in which the system equations are solved. We multiply a supplementary variate $i+1, j+1$, and $k+1$ and then sum over both $i, j$, and $k$. As a result, we obtained simultaneous equations for the system size distribution expressed in terms of the conditional expectations of the supplementary variables. We believe that our approach will help the readers better understand the discrete-time queueing systems and gain new insight into analyses of these systems.

\section{Appendix}

\section{A. Derivation of the Results in (4a) and (4b)}

We first sum the first equation in (3a), both over $i$ and $j, 0 \leq$ $i, j \leq \infty$; we have

$$
\sum_{i, j=0}^{\infty} Q_{0}(i, j)=\sum_{i, j=0}^{\infty} Q_{0}(i+1, j+1)
$$




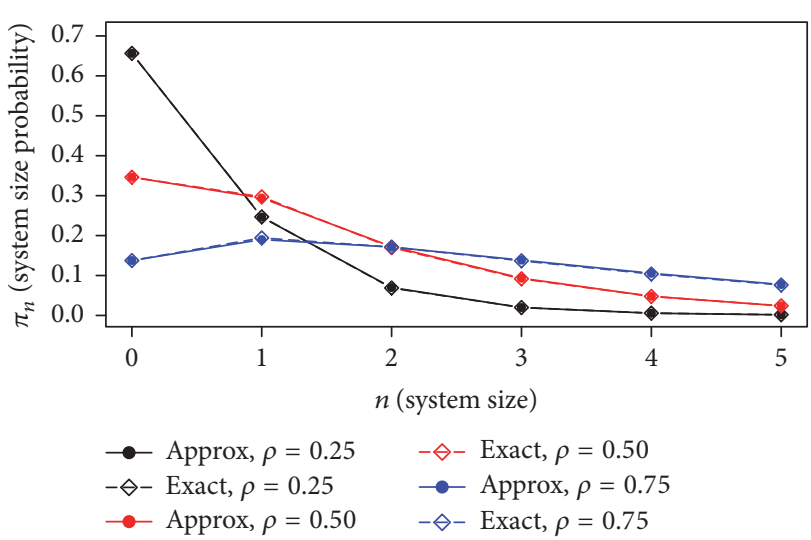

(a) $\mathrm{Geo} / \mathrm{MGeO}_{2} / 1 / \mathrm{GSV}$ queue

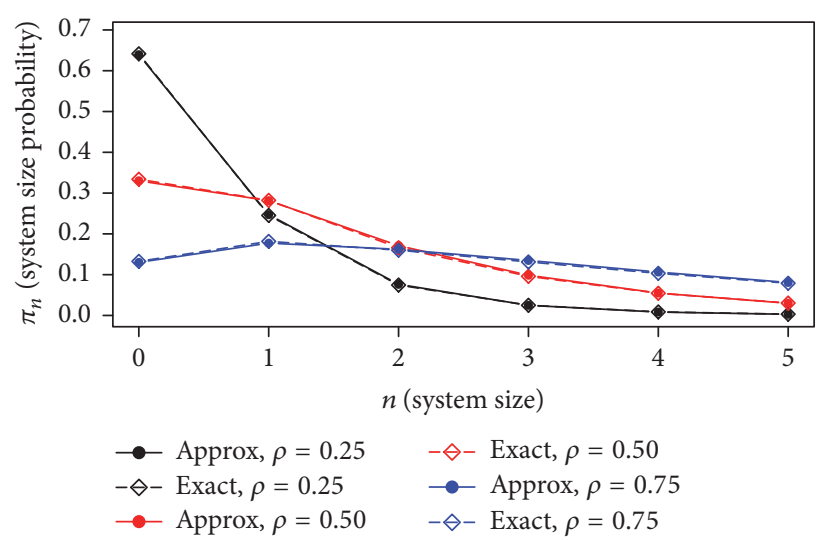

(b) $\mathrm{Geo} / \mathrm{MGeO}_{2} / 1 / \mathrm{MGeO}_{2} \mathrm{SV}$ queue

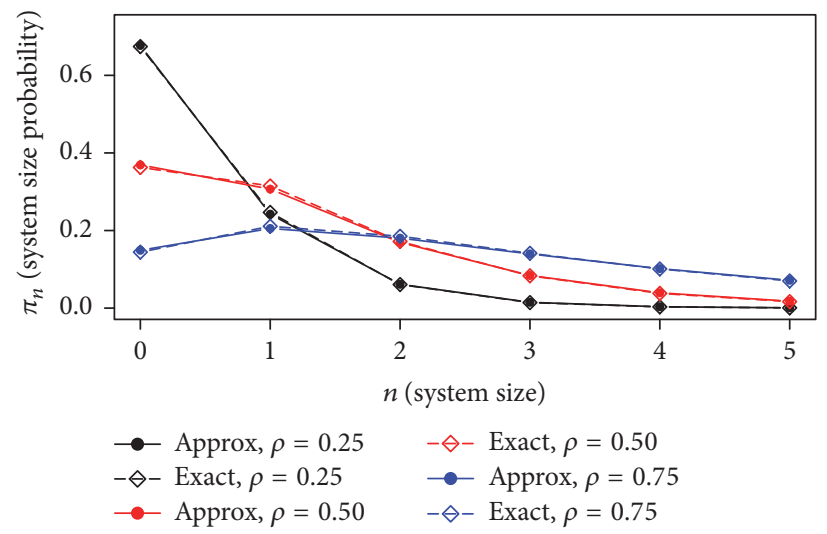

(c) $\mathrm{Geo} / \mathrm{MGeO}_{2} / 1 / \mathrm{NBSV}$ queue

Figure 2: Approximation of $\pi_{n}$ for $\mathrm{Geo} / \mathrm{MGeo}_{2} / 1 / \mathrm{SV}$ queue when $E[S]=2.5$ and $E[V]=5$.

$$
+\sum_{i=0}^{\infty} P_{1}(i+1,0) \sum_{j=0}^{\infty} v_{j+1}
$$

The left hand side of (A.1) is split into four terms as follows:

$$
Q_{0}(0,0)+\sum_{i=1}^{\infty} Q_{0}(i, 0)+\sum_{j=1}^{\infty} Q_{0}(0, j)+\sum_{i, j=1}^{\infty} Q_{0}(i, j)
$$

The right hand side of (A.1) is simplified as follows:

$$
\sum_{i, j=1}^{\infty} Q_{0}(i, j)+\sum_{i=1}^{\infty} P_{1}(i, 0) \text {. }
$$

Thus, we have

$$
\sum_{i=1}^{\infty} P_{1}(i, 0)=Q_{0}(0,0)+\sum_{i=1}^{\infty} Q_{0}(i, 0)+\sum_{j=1}^{\infty} Q_{0}(0, j) .
$$

Applying the same procedure to the rest of (3a), (3b), and (3c), we get the following relations:

$$
\begin{aligned}
& \sum_{j=1}^{\infty} Q_{n-1}(0, j) \\
& \quad=Q_{n}(0,0)+\sum_{i=1}^{\infty} Q_{n}(i, 0)+\sum_{j=1}^{\infty} Q_{n}(0, j), \quad n \geq 1,
\end{aligned}
$$

$$
\begin{aligned}
& P_{0}(0)=\sum_{i=1}^{\infty} Q_{0}(i, 0) \\
& \sum_{j=1}^{\infty} Q_{0}(0, j)+\sum_{k=1}^{\infty} P_{1}(0, k)=\sum_{i=1}^{\infty} Q_{1}(i, 0)+\sum_{i=1}^{\infty} P_{2}(i, 0) \\
& \sum_{i=1}^{\infty} P_{n}(i, 0)+\sum_{k=1}^{\infty} P_{n}(0, k) \\
& =Q_{n-1}(0,0)+\sum_{i=1}^{\infty} Q_{n}(i, 0)+\sum_{k=1}^{\infty} P_{n-1}(0, k) \\
& \quad+\sum_{i=1}^{\infty} P_{n+1}(i, 0), \quad n \geq 2 .
\end{aligned}
$$

By recursively solving (A.4) and (A.5) and simplifying them, we finally get the results in (4a) and (4b).

\section{B. Derivation of (11a), (11b), (11c), (11d), (11e), and (11f)}

Derivation of (11a), (11b), (11c), (11d), (11e), and (11f) is based on the definition of a conditional expectation. The proof of 


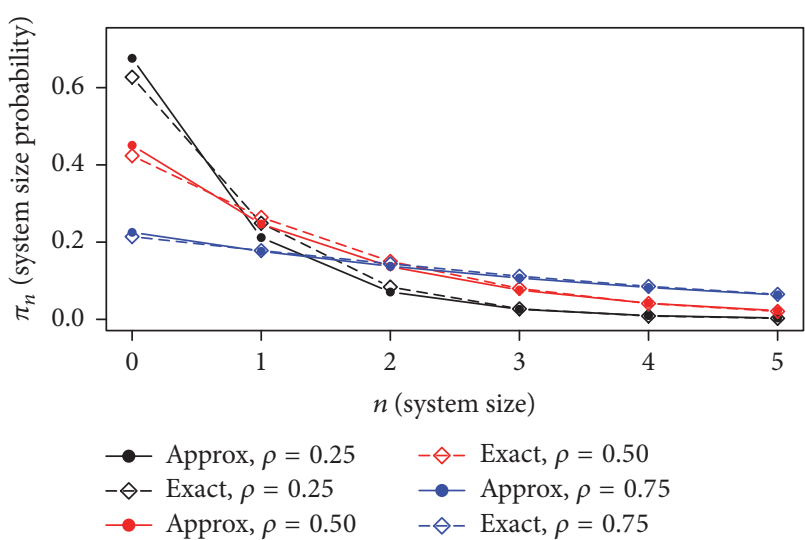

(a) $\mathrm{MGeO}_{2} / \mathrm{Geo} / 1 / \mathrm{GSV}$ queue

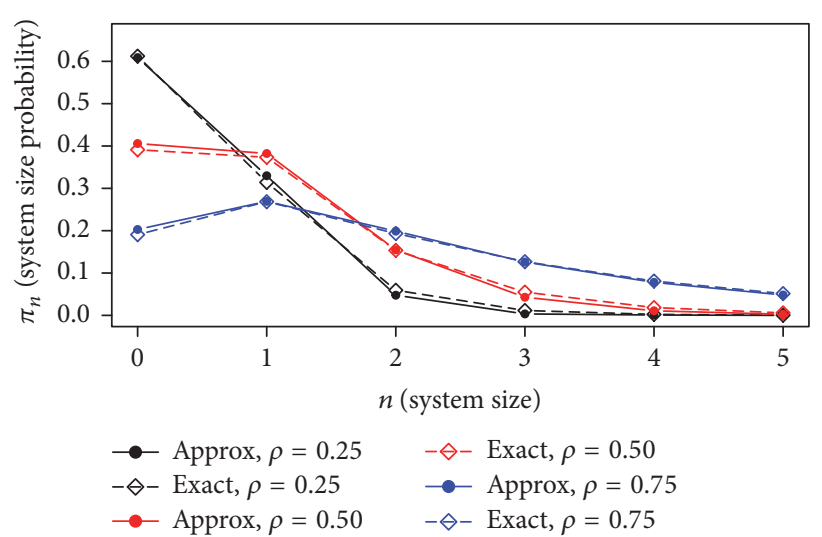

(b) $\mathrm{NB} / \mathrm{Geo} / 1 / \mathrm{GSV}$ queue

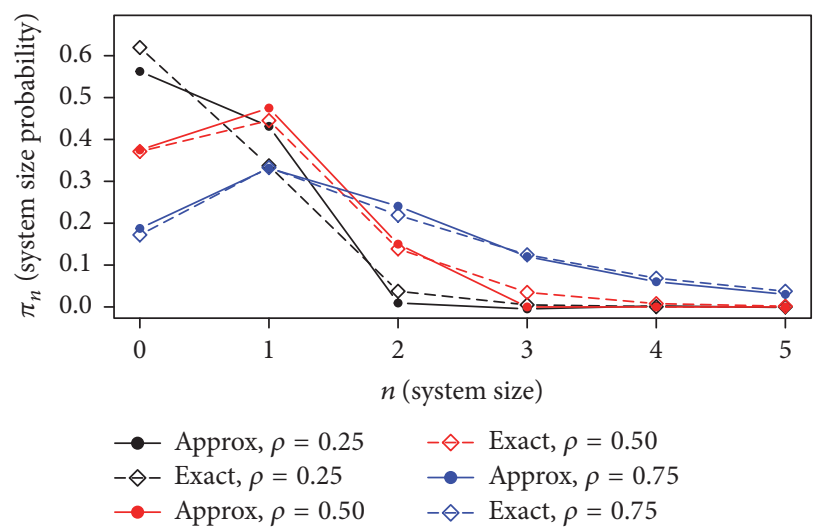

(c) Pois/Geo/1/GSV queue

FIGURE 3: Approximation of $\pi_{n}$ for GI/Geo/1/GSV queue when $E[A]=10$ and $E[V]=5$.

(11a), (11b), (11c), (11d), (11e), and (11f) centers on $\sigma_{n}$, which is the expected value of the remaining service time at the arrival epoch of a customer who sees $n$ customers in the system. Since the remaining service time at a customer arrival epoch contains 0 , we have

$$
\begin{aligned}
\sigma_{n} & =\lim _{t \rightarrow \infty} E\left[S_{R}\left(t^{-}\right) \mid N\left(t^{-}\right)=n, \xi\left(t^{-}\right)=1, A_{R}\left(t^{-}\right)=0\right] \\
& =\lim _{t \rightarrow \infty} \sum_{k=0}^{\infty} k \operatorname{Pr}\left\{S_{R}\left(t^{-}\right)=k \mid N\left(t^{-}\right)=n, \xi\left(t^{-}\right)=1, A_{R}\left(t^{-}\right)=0\right\} \\
& =\frac{\sum_{k=0}^{\infty} k \lim _{t \rightarrow \infty} \operatorname{Pr}\left\{N\left(t^{-}\right)=n, \xi\left(t^{-}\right)=1, A_{R}\left(t^{-}\right)=0, S_{R}\left(t^{-}\right)=k\right\}}{\lim _{t \rightarrow \infty} \operatorname{Pr}\left\{N\left(t^{-}\right)=n, A_{R}\left(t^{-}\right)=0, \xi\left(t^{-}\right)=1\right\}}=\frac{\sum_{k=0}^{\infty} k P_{n}(0, k)}{\sum_{k=0}^{\infty} P_{n}(0, k)}, n \geq 0 .
\end{aligned}
$$

From (5c), substituting $\lambda P_{n}^{A}=\sum_{k=0}^{\infty} P_{n}(0, k)$ into (B.1) completes the proof. One can make proofs of the other quantities in (11a), (11b), (11c), (11d), (11e), and (11f) through the above same procedures.

\section{Competing Interests}

The author declares that there is no conflict of interests regarding the publication of this paper.

\section{References}

[1] B. T. Doshi, "Queueing systems with vacations-a survey," Queueing Systems, vol. 1, no. 1, pp. 29-66, 1986.

[2] H. Takagi, Queueing Analysis, Vol. 2: Finite Systems, NorthHolland, Amsterdam, The Netherlands, 1991.

[3] H. Takagi, Queueing Analysis, Volume 3: Discrete-Time Systems, North-Holland Publishing, Amsterdam, The Netherlands, 1991. 
[4] K. C. Chae, S. M. Lee, and S. H. Lee, "The discrete-time GI/Geo/1 queue with single geometric vacation," Quality Technology \& Quantitative Management, vol. 5, no. 1, pp. 21-31, 2016.

[5] D. Fiems and H. Bruneel, "Analysis of a discrete-time queueing system with timed vacations," Queueing Systems. Theory and Applications, vol. 42, no. 3, pp. 243-254, 2002.

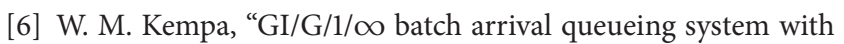
a single exponential vacation," Mathematical Methods of Operations Research, vol. 69, no. 1, pp. 81-97, 2009.

[7] K. C. Chae, N. K. Kim, and B. K. Yoon, "On the queue length distribution for the $\mathrm{GI} / \mathrm{G} / 1 / \mathrm{K} / \mathrm{V}_{M}$ queue," Stochastic Analysis and Applications, vol. 22, no. 3, pp. 647-656, 2004.

[8] D. W. Choi, N. K. Kim, and K. C. Chae, "A two-moment approximation for the GI/G/c queue with finite capacity," INFORMS Journal on Computing, vol. 17, no. 1, pp. 75-81, 2005.

[9] N. K. Kim and K. C. Chae, "Transform-free analysis of the GI/G/ $1 /$ K queue through the decomposed Little's formula," Computers and Operations Research, vol. 30, no. 3, pp. 353-365, 2003.

[10] S. M. Ross, Stochastic Processes, John Wiley \& Sons, New York, NY, USA, 2nd edition, 1996. 


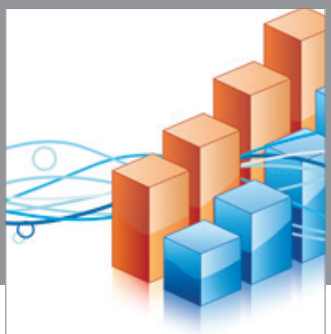

Advances in

Operations Research

vatem alat4

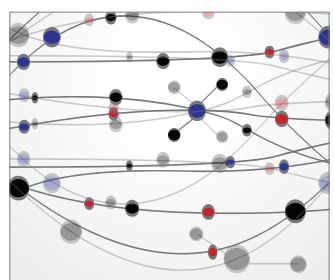

\section{The Scientific} World Journal
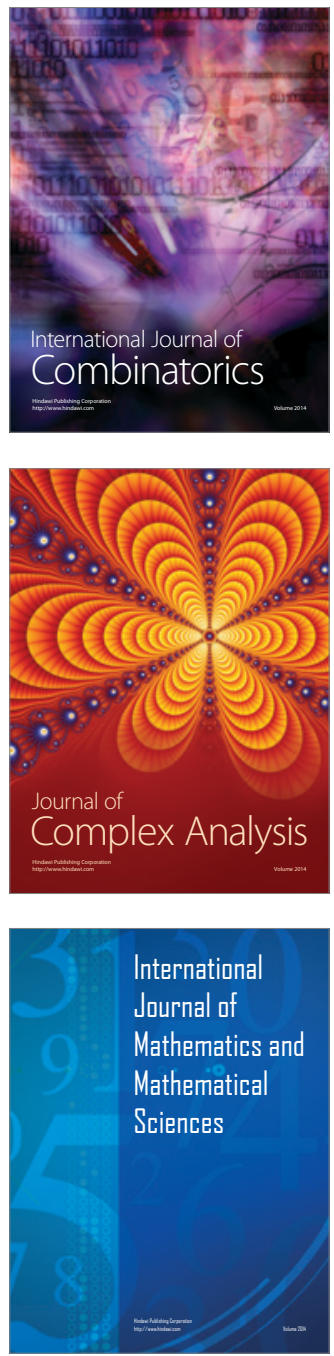
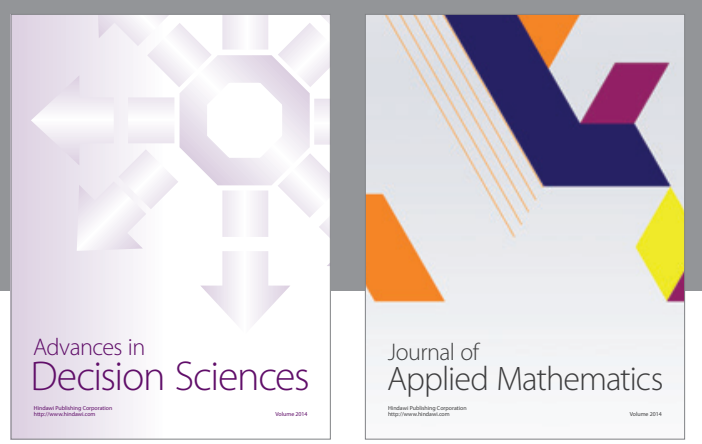

Algebra

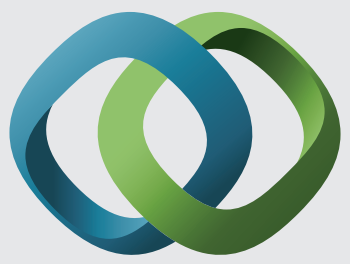

\section{Hindawi}

Submit your manuscripts at

http://www.hindawi.com
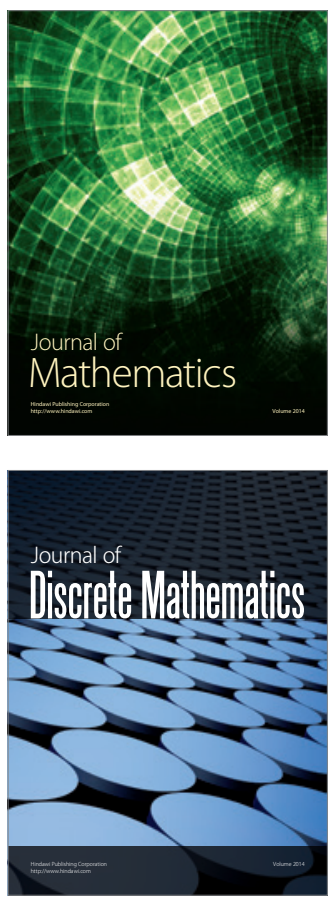

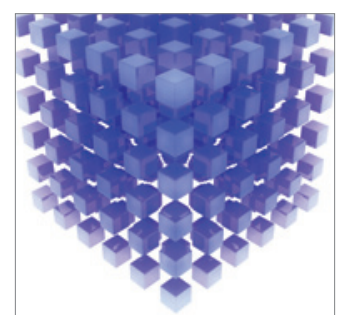

Mathematical Problems in Engineering
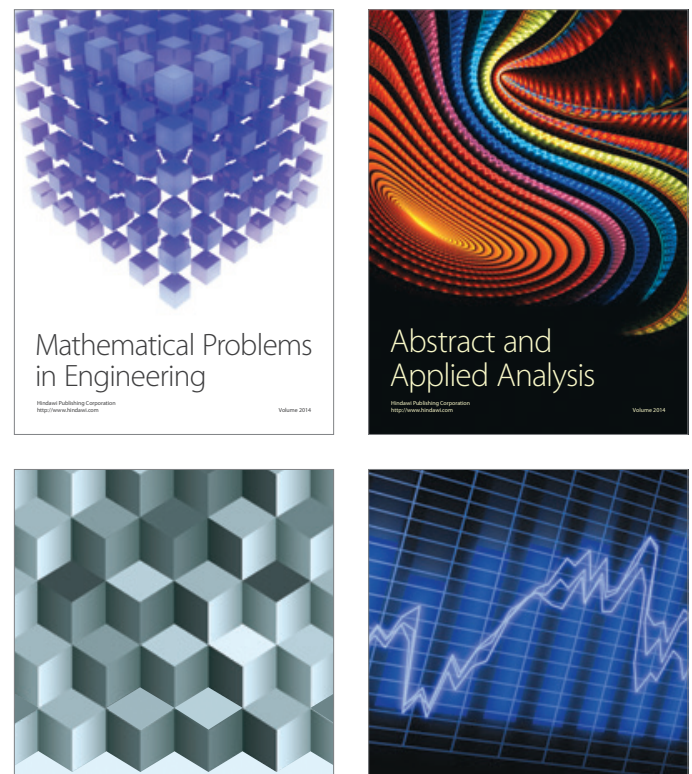

Journal of

Function Spaces

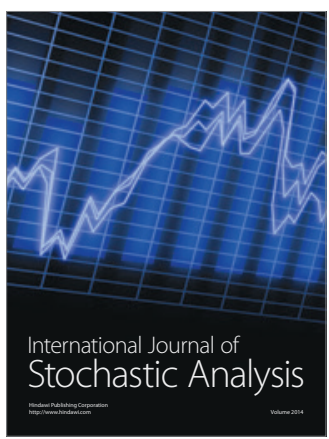

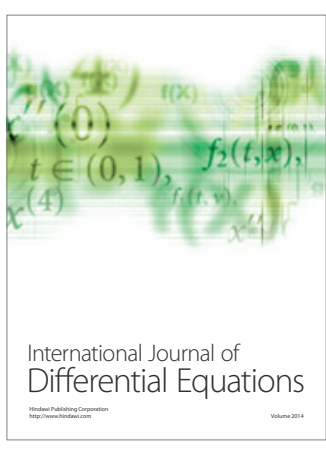
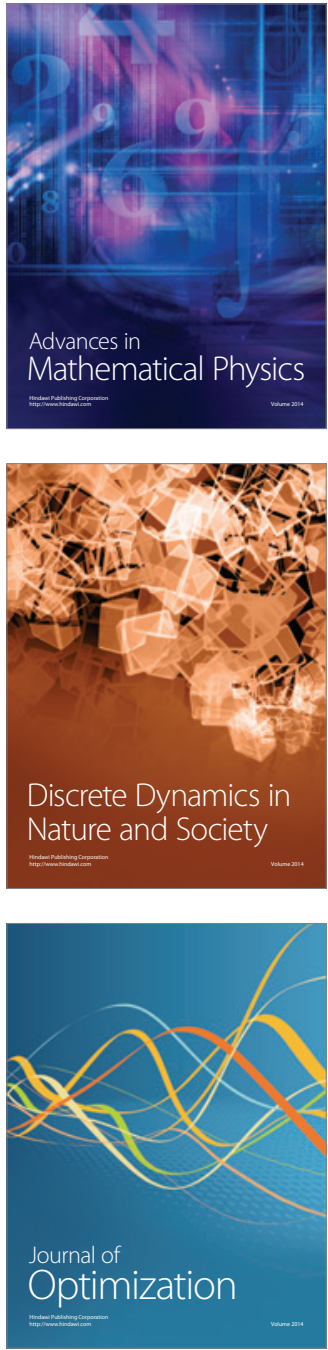